\title{
DEVELOPMENT OF ENERGY SYSTEMS PLANNING METHODS
}

\author{
Nikolay Belyaev ${ }^{1, *}$, Andrey Egorov ${ }^{1}$, Nikolay Korovkin ${ }^{2}$ and Vladimir Chudny ${ }^{2}$ \\ ${ }^{1}$ JSC «Technical Inspection UES», Russia \\ ${ }^{2}$ Peter the Great St.Petersburg Polytechnic University, Russia
}

\begin{abstract}
The article is devoted to improving the methods of planning the development of an electric power system (hereinafter - EPS) for the long term. The list of tasks to be solved when planning the development of EPS, the formulation of the task of substantiating the development of EPS, and the features of its solution are considered. Within the framework of the study of the multicriteria formulation of the problem of substantiating the development of EPS, possible criteria for planning an EPS are proposed. Calculations were performed for two and four criteria. Based on the results obtained, conclusions were drawn about the prospects of a multicriteria approach to planning the development of EPS.
\end{abstract}

\section{Introduction}

Current conditions of electric power development in the world feature increasingly stringent requirements for EPS safety and quality of power supply to consumers, which is due, on the one hand, to economically reasonable trends dealing with deepening electrification of the economy and households (including the introduction of modern production technologies and digitalization of technological processes), on the other hand, to the growth of social and economic importance of reliable electric power supply, especially in big and metropolitan cities. In this case, strong restrictions are imposed on the development of energy engineering in respect of price and tariff consequences of adopted investment decisions that are also due to socio-economic factors expressed in restraining the increase of electricity and demand capacity prices.

Along with these trends, the development of the electric power industry is characterized by the active introduction of new technologies, such as flexible AC transmission systems (FACTS), high-capacity energy storage, and demand Response management, allow for enhancing significantly the EPS efficiency, while reducing capital and operating costs.

Under such conditions arises the problem of increasing the efficiency of power system planning for the purposes of cost minimization so as to ensure the growing energy demand while meeting eligible technical, environmental and economic requirements with account made for the implementation of mentioned advanced technologies. Given the fact that modern EPS are large power units that may include tens of thousands of units of generating equipment, power transmission lines and substations, the task of EPS planning is generally reduced to a multi-criteria optimization problem to be solved on a discrete set of higher dimensions. The solution of such problems is only possible with modern capabilities of computer technology, but still requires the development of special methods.

\section{The modern practice of energy system planning}

To date, the main methodological provisions for planning the development of EPS have been thoroughly worked out and set out in both domestic and foreign publications. In general, these methodological provisions were developed and generalized during the period of the centralized development of EPS [1,2] and received additional development in connection with the reform and decentralization of management in the electric power industry in recent decades $[3,4]$. The indicated methodological provisions were used in the development of normative and technical documents governing the planning and design of EPS development, as well as specialized software and computer systems. The structure of tasks to be solved in planning the development of EPS can be summarized in the form of several stages:

1. Forecasting the development of technologies for the production, transmission, accumulation, and distribution of electrical energy.

2. Forecasting the demand for electricity and capacity.

3. Substantiation of the rational structure of generating capacities.

4. Justification of the location and composition of generating capacities.

5. Justification for the development of the main (backbone) electrical network.

6. Assessment of the economic and environmental consequences of the development of the electric power industry.

\footnotetext{
Corresponding author: Belyaev.NA@yandex.ru
} 
Stages 1 and 2 relate to forecasting the external conditions of EPS functioning for the future. Stages 3 - 6 relate directly to the justification of the development of EPS and are carried out sequentially, while at each stage of planning, the decisions obtained in the previous stages are refined.

The standard problem statement of the growth substantiation EPS reduces to a cost-minimizing procedure in relation to total reduced costs 3 connected with the electric power supply to consumers [2]:

$$
\begin{aligned}
& C(\mathbf{x})=\sum_{t}\left(C_{t}^{\mathrm{K}}(\mathbf{x})+C_{t}^{\mathrm{O}}(\mathbf{x})\right) \cdot(1+d)^{-t} \underset{\mathbf{x}}{\longrightarrow} \text { min, } \\
& \mathbf{x} \in R,
\end{aligned}
$$

where $C_{t}^{\mathrm{K}}, C_{t}^{\mathrm{O}}$ - capital and operational costs per year $t$ respectively, $d$-discount rate.

Variables $\mathbf{x}$ in problem (1) are the actions taken for the development of systems for the production, transmission, and distribution of electrical energy, which determine the amount and structure of costs. These activities include:

- modernization of existing generating capacities, including actions to extend their resource;

- construction of new generating units (power units) at existing or new power plants of various types;

- buildup of electric energy storage units, including pumped storage power plants;

- buildup of new power lines;

- increasing the transformer capacity of substations;

- actions to increase the transmission capacity of the electrical network, including the use of reactive power compensation means or FACTS devices, and others.

Each action is characterized by technical and economic indicators, including the amount of capital and operating costs.

The area of limitations $R$ is determined by the requirements for the reliability and safety of EPS that affect the choice of individual technical solutions or their combinations. These requirements include both technological restrictions on the operation modes of the EPS equipment and restrictions on the operation of the EPS as a whole. The first group of restrictions includes restrictions on a load of generating equipment - these are available capacity, technological minimum, integral restrictions on electricity generation, as well as power grid equipment - these are permissible current loading and voltage levels, maximum power fluxes under stability conditions. The second group includes the required level of EPS reliability, the permissible impact of electric power facilities on the environment, the available investment, and others.

Statement (1) contains the principle of minimizing the costs of power supply to the economy and households while observing the mandatory requirements for the operation of EPS. Taking into account the discreteness of measures for the development of EPS, for modern large EPSs, the solution of problem (1) is very laborious and comes down to finding the global optimum on a representative discrete set. In this regard, in practice, when solving (1), a number of assumptions are used, including the decomposition of the problem (1) into the above stages and the use of a simplified (aggregated) representation of the EPS structure at the initial stages of the solution. This makes it possible to reduce the dimension of the problems solved at each stage and to use standard optimization methods, including linear programming methods, to solve them. This, however, requires refinement of the obtained solution at subsequent stages, including compliance with the specified constraints and, in general, reduces the accuracy of the solution (1).

In addition to the computational complexity, the problem of substantiating the development of EPS in the formulation (1) is accompanied by two methodological problems. The first problem concerns the tasks of planning the development of EPS and lies in the uncertainty of a number of initial data, including external conditions for the functioning of EPS. These are the demand for electricity and capacity, fuel prices, etc. Also, the technical and economic indicators of certain measures for the development of EPS are poorly defined. These are cost indicators, reliability indicators, etc. Uncertainty of the initial data, especially in the longterm planning of EPS, led to the introduction of probabilistic methods for planning the development of EPS, widely presented in [5].

The second problem, for the statement (1), is the complexity of describing the region of limitations $R$. The limitations of the first group, associated with the operating modes of the EPS equipment, are determined, as a rule, unambiguously. However, the restrictions of the second group, imposed on the functioning of the EPS as a whole, in some cases are difficult to set with fixed values of the corresponding indicators. Such limitations, for example, include the required level of EPS reliability. Theoretically, the required level of EPS reliability is determined by a separate feasibility study, taking into account the costs of ensuring reliability (redundancy) and compensation for damage from unreliability (interruptions in power supply and shortage of electricity). In practice, such an approach is unrealizable due to the variety of EPS consumers and the lack of unambiguous estimates of the specified damage. Similarly, it is difficult to unambiguously determine the restrictions on the impact of EPS on the environment, since such restrictions are set for the industry as a whole, and not only for electric power facilities. In this regard, some of the constraints that form the domain $\mathrm{R}$ in problem (1), it is advisable to transfer into additional functionals. Thus, problem (1) is transformed into a multicriteria problem. The main approaches to solving multicriteria problems of substantiating the development of EPS are considered in [4].

\section{Development of energy system planning methods}

Taking into account the increasing requirements for the functioning of power systems and the complexity of the unambiguous definition of a number of restrictions, which as noted above, it seems promising for the development of the theory of planning EPS is the transition to multi-criteria formulations of the problem of 
substantiating the development of EPS. The following can be taken as possible criteria for justifying the development of EPS:

- criteria for the reliability of the EPS functioning, including the functions of the expected shortage of electrical energy and power;

- criteria for the economic efficiency of EPS, including criteria for the social efficiency of the development of the electric power industry and the commercial efficiency of individual projects in it;

- criteria for the reliability and quality of power supply to consumers, including the functions of the expected frequency and duration of power supply interruptions;

- criteria for the impact of EPS facilities on the environment, including emissions of pollutants by power plants operating on fossil fuel;

- criteria for energy security, including criteria reflecting the diversification of the fuel and energy balance and redundancy of critical EPS facilities;

other criteria reflecting the efficiency of EPS.

Modern methods of multicriteria optimization, including those applied to solving problems in the electric power industry, are known [6]. From a formal point of view, in multicriteria optimization, it is of interest to rank the set of Pareto optimal solutions obtained from the optimization results and to select specific solutions for their subsequent implementation. Currently, the approaches used for this [4] are, in one way or another, based on expert assessments, with the use of which, for example, ranking criteria by significance or assigning weight coefficients to them is performed.

The second promising direction in the theory of EPS planning is the use of modern evolutionary optimization algorithms, for example, the genetic algorithm, ABC, or BAT algorithms, which are widely used to solve discrete global optimization problems, including in related industries [7,8]. These algorithms use directed enumeration and thus are well combined with probabilistic methods for planning the development of EPS, which currently use random enumeration (methods of the Monte Carlo type). It is expected that the use of these algorithms will significantly increase the efficiency of justifying the development of an EPS, including allowing one to abandon the assumptions made in solving (1).

Next, we will consider the results of solving several multicriteria optimization problems obtained using a genetic algorithm when planning the development of an EPS.

For EPS with a maximum electrical load of $13 \mathrm{GW}$, the structure of generating capacities was optimized according to two criteria:

$$
\begin{aligned}
& C(\mathbf{x}) \longrightarrow \mathbf{x} \min , \\
& J_{D}(\mathbf{x}) \stackrel{ }{\longrightarrow} \min , \\
& \mathbf{x} \in R .
\end{aligned}
$$

where $C$ is the total reduced costs for the construction and operation of generating capacities, $J_{D}$ is the probability of a power shortage in the EPS [9], $\mathrm{R}$ is the area of limitations reflecting the permissible operating modes of the generating equipment.

When optimizing, various types of generating equipment were considered, including nuclear and hydroelectric power plants, units of thermal power plants of various types and unit capacity. Technical and economic indicators and indicators of the reliability of generating equipment are taken according to reference data. The results of the solution (2) are presented in Fig. 1 .

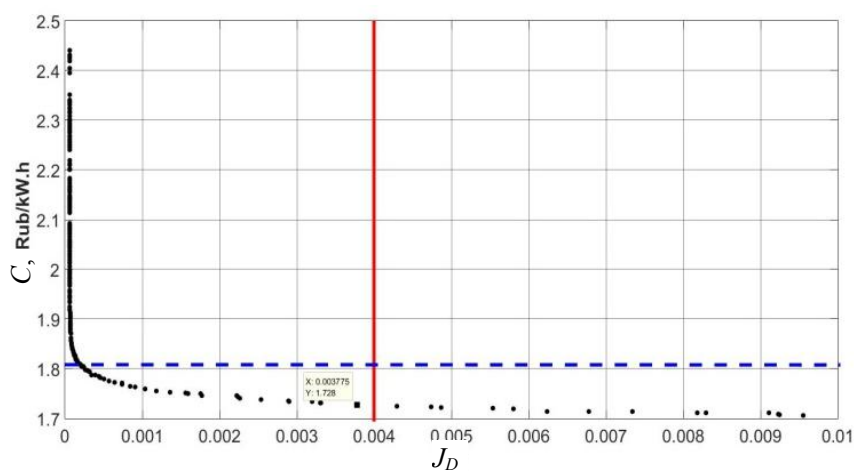

Fig. 1. Results of optimization of the structure of generating capacities according to two criteria.

On Fig. 1 the values of target functions (2) are plotted on axes: on horizontal line - probability of capacity shortage, on vertical line - working cost of electric energy compatible with accumulated total costs. Points mark the results. The total number of obtained solutions is equal to 375 [10].

During Soviet time the regulated value of CAI has been justified and until now it is applied at the level of 0,996 , that is in line with $J_{D}=0,004$. Thus, when using this rate one should choose the solution marked on Fig. 1 , in this case the electricity cost will make 1,73 Rub./kWh. However this option does not seem to be optimal if other solutions are examined. For example, an insignificant price increase within $1 \mathrm{cop} . / \mathrm{kWh}$ allows reducing $J_{D}$ to the level of 0,00226 . Moreover, it should be noted that from cause-and-effect view under circumstances when the operating conditions of EPS through the long run are uncertain, the parameter setting error for technologies applied in problem as source data, cost parameters, first of all, may reach up to 5 per cent and more that significantly affects the accuracy of cost assessment. In this case the price increase by 5 per cent as compared to the solution marked on Fig. 1 (up to 1,81 Rub./kWh, shown by horizontal dot lines) allows reducing $J_{D}$ up to 0,00016 , i.e. more than by an order of magnitude.

Further reduction of $J_{D}$ may appear to be costineffective as costs progress at a far quicker rate and an insignificant reduction of $J_{D}$ results in price hikes. At a price of $1,97 \mathrm{Rub} . / \mathrm{kW} \cdot \mathrm{h}$ the limit value of $J_{D} \sim 6,3 \cdot 10^{-5}$, is reached, in practice it is insensitive to price increase.

The range of installed capacity variation upon got solutions was within $14,95 \mathrm{GW}-22,15 \mathrm{GW}$ that is in line with the margin capacity value from $16,2 \%$ to $72,1 \%$ of EPS maximum load. For the solution marked on Fig. 1 which is in conformity with regulated CAI, the capacity margin was $19,7 \%$. For the solution with the 
price of 1,81 Rub. $/ \mathrm{kW}$ and $J_{D}=0,00016$ the capacity margin is equal to $29,8 \%$.

In Fig. 2 shows the results of optimization of the structure of generating capacities of EPS with the same initial conditions according to four criteria:

$$
\begin{aligned}
& C(\mathbf{x}) \stackrel{\mathbf{x}}{\longrightarrow} \min , \\
& J_{D}(\mathbf{x}) \stackrel{\mathbf{x}}{\longrightarrow} \min , \\
& W_{F}(\mathbf{x}) \stackrel{\mathbf{x}}{\longrightarrow} \min , \\
& P_{M A X}(\mathbf{x}) \stackrel{\mathbf{x}}{\longrightarrow} \min , \\
& \mathbf{x} \in R .
\end{aligned}
$$

where $W_{F}$ is the share of electricity generated at thermal power plants, $P_{M A X}$ is the maximum share of the type of generating capacity in the structure of installed capacity.

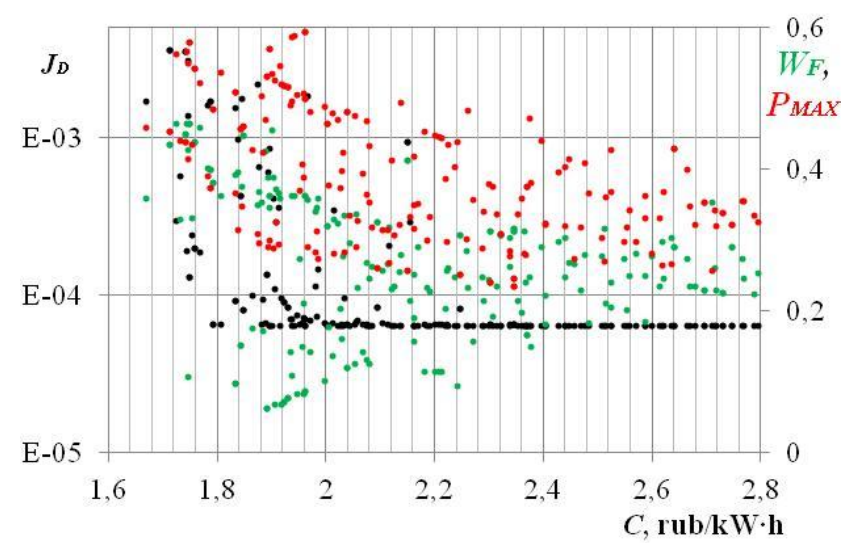

Fig. 2. Results of optimization of the structure of generating capacities according to four criteria.

On Fig. 2, along the abscissa axis is shown the cost value, the ordinate axis is shown the probability of a power shortage (black marker), and the additional ordinate axis are shown the $W_{F}$ and $P_{M A X}$ values (green and red markers, respectively).

From the presented results, it can be seen that the minimum level of the probability of a power shortage is achieved at a cost of 2 Rubles / $\mathrm{kWh}$. This corresponds to the obtained solution (2). At the same time, decisions with a cost level within 2 Rubles / $\mathrm{kWh}$ are characterized by a conflict of two other criteria. The so-called "green" solutions (with a $W_{F}$ value of less than 0.2 ) have high $P_{M A X}$ values, namely, a high share of nuclear power plants in the structure of generating capacities. On the contrary, solutions with relatively low $P_{M A X}$ values are characterized by high $W_{F}$ values (at the level of 0.3 0.5 ). To obtain a more favorable combination of the values of these criteria, it is necessary to consider solutions with a higher level of costs, which provide for the construction of hydroelectric and power plants on renewable sources. This means that if the last two criteria are set in problem (3) as constraints, depending on the choice of their values, solutions in the low-cost area can be cut off and excluded from consideration.

\section{Conclusion}

The growing requirements for the technological and economic efficiency of EPS, the technological development of the electric power industry requires an increase in the accuracy and efficiency of planning and justification of EPS development. Taking into account the general formulation of the problem of justifying the development of EPS, a promising method for its solution is the use of multicriteria optimization using modern evolutionary algorithms.

As the calculations show, the translation of a number of restrictions into criteria expands the search area for solutions for development EPS. This makes it possible not only to take into account the regulatory requirements for the values of certain criteria but also to choose the optimal combination of the considered criteria, taking into account the structure of a specific EPS and possible measures for its development.

\section{References}

1. L.S.Belyaev, G.V.Voytsehovskaya, V.A.Saveliev and others, A systematic approach to managing the development of the electric power industry (Nauka, Novosibirsk, 1980)

2. I.M.Volkenau, A.N.Zeiliger, L.D.Khabachev, Economics of electric power system formation (Energiya, Moscow, 1981)

3. A.A.Makarov, F.V.Veselov, Ye.A.Volkova, A.S.Makarova, Methodological bases of development of electric power industry development prospects (ERI RAS, Moscow, 2007)

4. N.I.Voropai, S.V.Podkovalnikov, V.V.Trufanov and others, Justification for the development of electric power systems: Methodology, models, methods, and their use (ERI RAS, Moscow, 2007)

5. W.Li, Probabilistic transmission system planning (IEEE, EIC BC Hydro, 2011)

6. N.V.Korovkin, M.V.Odintsov, O.V.Frolov, Power Technology and Engineering, E 1, 75 (2016)

7. N.V.Korovkin, S.L.Gulay, D.A.Verkhovtcev, Bulletin of the RAS: Energy, E 4, 42 (2019)

8. E.Solovyeva, 2017 20th Conference of Open Innovations Association FRUCT (FRUCT20), DOI: 10.23919/FRUCT.2017.8071343 (2017)

9. D.Krupenev, G.Kovalev, L.Lebedeva, E3S Web of Conferences, 139, 01019 (2019)

10. N.Belyaev, A.Egorov, N.Korovkin, V.Chudny, E3S Web of Conferences, 139, 01004 (2019) 